\title{
Baryonic Tully-Fisher relation and star formation rate
}

Masato I.N. Kobayashi*

Nagoya University

E-mail: kobayashi.masato@d.mbox.nagoya-u.ac.jp

Tsutomu T. Takeuchi

Nagoya University

E-mail: takeuchi.tsutomu@g.mbox.nagoya-u.ac.jp

We investigated the relation between rotational velocity, baryonic mass, and star formation rate (SFR) for several samples of galaxies, and found a fundamental plane in a 3-dim space on which those galaxies are located.

In order to understand the galaxy evolution, we first need to investigate the physical properties of galaxies. Earlier research have shown the existence of scaling laws that connect two physical properties of galaxies, such as the Tully-Fisher relation, the baryonic Tully-Fisher relation, the star formation main sequence, the Schmidt-Kennicutt law, and so on. However, up to now they are treated separately. Therefore, when it comes to understanding and determining the galaxy evolution for each galaxy, it is essential to integrate those scaling laws to construct a unified fundamental property that explains the galaxy evolution universally in terms of their physical properties. In this study, we compiled observational data for 96 galaxies to find a fundamental plane in a 3-dim space. We interpret that this plane is one projected aspect of the unified fundamental relation that resides in much higher dimensions spanned by more physical properties. This would be the first step to investigate what properties are essential towards etablishing a unified theory.

The Life Cycle of Dust in the Universe: Observations, Theory, and Laboratory Experiments 18-22 November, 2013

Taipei, Taiwan

\footnotetext{
* Speaker.
} 


\section{Introduction}

To understand the galaxy evolution, it is one of the most important tasks to reveal the relation between galaxies and dark halos, because galaxies form and evolve in these dark halos' potential. In the history of the Universe, dark haloes formed first and merged with each other with the passage of time, and the first stars were born inside these dark halos. Later galaxies formed there and evolved.

The Tully-Fisher relation ([1]: TFR) is an empirical relation between the galaxy rotational velocity and its luminosity. This relation works effectively to determine the distance to relatively nearby galaxies and also works as an important relation to connect baryons and dark matter of a galaxy. On the one hand, the stars contained in a galaxy contribute to its luminosity; on the other hand, the dynamical mass, which is the sum of baryonic mass and dark matter mass of a galaxy, generates the rotational velocity. Thus, the tightness in the TFR implies there is a strong relation between baryons and dark matter. Although the TFR is a well-established linear relation, it had been known that low luminosity galaxies are deviated donward from this tight power-law relation.

Reference. [2] discovered that this power-law relation is restored if we employ baryonic mass instead of luminosity (baryonic mass is the sum of stellar mass and gas mass of a galaxy). This power-law relation is called the baryonic Tully-Fisher relation (BTFR). BTFR indicates baryonic mass is more fundamental property than luminosity because low luminosity galaxies contain large amount of gas that is still not turned into stars and do not create stars as effectively as bright galaxies do. Since the rotational velocity is generated from the potential of dynamical mass, BTFR is a strong standpoint to investigate the galaxy evolution in the sense of dark matter and baryon interaction in general.

Another intersting empirical relation of galaxies is star-formation main sequence (SFMS). Star-forming galaxies gather and get aligned to make this prominent sequence on the two dimensional plane spanned by stellar mass $\left(M_{*}\right)$ and star formation rate (SFR). Here, SFR is a rate at which a galaxy creates stars in the unit of $\mathrm{M}_{\odot} \mathrm{yr}^{-1}$, and $M_{*}$ is the galaxy's stellar mass.

If only dark matter were involved in the galaxy evolution, the related physics would be relatively simple because dark matter only interacts gravitationally. However, the active evolution includes baryons that involve a wide range of physical processes to make this evolution complicated. Thus, the analysis of galaxy evolution cannot stand without research of the baryonic properties. In this context, the SFMS is an star-forming aspect of these baryonic properties and represents the star formation history of a galaxy. Then, we expected that a similar relation would also exist between SFR and baryonic mass because stars are created from gas.

Although BTFR and SFMS seem to be clear, the physical justification for the reason why they are so tight is actually still under debate.

In this work, we considered these two empirical relations BTFR and SFMS to find fundamental properties behind all spiral galaxies.

\section{Data}

Our data consist of seven articles. [3], [4], [5], [6], [7], [8], and [9]. In total, we complied 96 galaxies out of the ones listed in the literature. 


\section{Result}

We extracted data of the rotaional velocity, stellar mass, and gas mass or HI mass from each article. Hereafter, we denote stellar mass as $M_{*}$, gas mass as $M_{\text {gas }}$, baryonic mass as $M_{\text {bar }}$, and rotational velocity as $V_{\text {rot }}$. If $M_{\text {gas }}$ and $M_{\text {bar }}$ are not available, we converted HI mass to gass mass by $M_{\mathrm{gas}}=1.4 M_{\mathrm{HI}}$. Then, baryonic mass can be obtained by $M_{\mathrm{bar}}=M_{*}+M_{\mathrm{gas}}$.

To determine the fundamental plane precisely, a homogenized SFR estimation method is desired. We especially need to take into account the dust attenuation of ultraviolet(UV)/opt photons and infrared (IR) re-emission from dust. This can be achieved by compiling observational data in both IR and UV for each galaxy. Now we are using far-IR (FIR) data collected by AKARI satellite (90 $\mu \mathrm{m}$ and $140 \mu \mathrm{m}$ ) to estimate star formation hidden by dust studied by [10]:

$$
\log \mathrm{SFR}_{\mathrm{dust}}=\log L_{\mathrm{TIR}}-9.75+\log (1-\eta)
$$

where $L_{\mathrm{TIR}}$ is the total IR luminosity estimated from the combination of flux densities in $90 \mu \mathrm{m}$ and $140 \mu \mathrm{m}$. Also $\eta$ represents a fraction in far-IR that is contributed from old stellar population. We follow [10] to apply the value $\eta=0.3$.

We analized the data by principal component analysis (PCA). From this procedures, we found the relation between these three properties in SFR- $M_{\mathrm{bar}}-V_{\text {rot }}$ space as follows:

$$
\log \mathrm{SFR}_{\text {dust }}=110 \log V_{\text {rot }}-26.8 \log M_{\text {bar }}-36.4 \pm 8.9
$$

This relation forms a 2-dim fundamental plane which subsumes all sample galaxies on its surface, which means this could underly all spiral galaxies and represents the relation between their dark matter, baryon, and star formation history.

\section{Discussion}

We showed that the galaxies are on a fundamental plane defined by Eq. 3.2. This relation has one significant feature. SFR is inversly proportional to $M_{\text {bar }}$. This makes us suspect that SFR decreases if baryonic mass increases, but that is not the case. Eq. 3.2 works as a constraint concerning three properties "simultaneously", thus what really happens

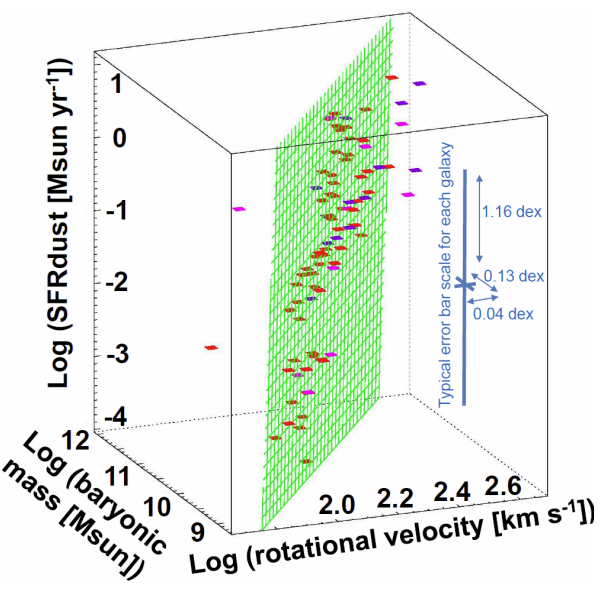

Figure 1: Fundamental plane constructed by 96 sample galaxies, which is illustrated by Eq. 3.2 is a galaxy that has larger baryonic mass has bigger rotational velocity. After the competition between the increments of baryonic mass and rotational velocity, this results in larger SFR.

\section{Future Prospects}

(1) We need to examine further the SFR dependece on $V_{\text {rot }}$ and $M_{\text {bar }}$ represented by Eq. 3.2 and follow up physical meaning in this relation. 
(2) To estimate SFR more precisely, we also need to combine observational data in ultraviolet. [10] lay out a method to combine FUV data with FIR data.

(3) It is a current trend to investigate how far we can extend the BTFR toward lower mass but very gaseous galaxies, thus we should also work on how far we can extend the fundamental plane in the three dimensions. Observational progress for low mass and slightly star-forming galaxies will be in demand. This is one of the big expectations on SKA.

(4) There is another attracting scalling law related to star formation: Schmidt-Kennicutt law (KSlaw). Since KS-law links two surface density quantities, radius will be the next appropriate property that we need to count when we incorporate KS-law into the three dimensional relation Eq. 3.2. This immediately commands you to handle higher dimensional manifold and involves multi-dimensional data analysis. Then, PCA works more powerfully than now. From this viewpoint, we declare that the fundamental relation we presented above is one aspect of the unified fundamental property existing as a manifold in much higher dimensions.

(5) To examine this fundamental relation in the context of not just property but evolution, we need to analyze the three dimensional relation at different redshifts and establish the extended BTFR.

\section{References}

[1] Tully, R. B., \& Fisher, J. R. 1977, A\&A, 54, 661

[2] McGaugh, S. S., Schombert, J. M., Bothun, G. D., \& de Blok, W. J. G. 2000, ApJ, 533, 99

[3] McGaugh, S. S. 2005, ApJ, 632, 859

[4] Gurovich, S., Freeman, K., Jerjen, H., Staveley-Smith, L., \& Puerari, I. 2010, AJ, 140, 663

[5] Torres-Flores, S., Epinat, B., Amram, P., Plana, H., \& Mendes de Oliveira, C. 2011, MNRAS, 416, 1936

[6] Brosch, N., Spector, O., \& Zitrin, A. 2011, MNRAS, 415, 431

[7] McGaugh, S. S. 2012, AJ, 143, 40

[8] Hall, M., Courteau, S., Dutton, A. A., McDonald, M., \& Zhu, Y. 2012, MNRAS, 425, 2741

[9] Torres-Flores, S., Mendes de Oliveira, C., Plana, H., Amram, P., \& Epinat, B. 2013, MNRAS, 432, 3085

[10] Takeuchi, T. T., Buat, V., Heinis, S., Giovannoli, E., Yuan, F.-T., Iglesias-Paramo, J., Murata, K. L., \& Burgarella, D. 2010, A\&A, 514, A4 